\title{
Combined machine learning and functional magnetic resonance imaging allows individualized prediction of high-altitude induced psychomotor impairment: The role of neural functionality in putamen and pallidum
}

\author{
Xiaoming Chen ${ }^{\S}$, Qian Zhang ${ }^{\S}$, Jiye Wang ${ }^{\S}$, Zhenlong Xin, Jingyuan Chen*, Wenjing Luo* \\ Department of Occupational and Environmental Health, the Ministry of Education Key Lab of Hazard Assessment and Control in \\ Special Operational Environment, School of Public Health, Air Force Medical University, Xi'an, Shaanxi Province, China.
}

\begin{abstract}
Summary Hypoxia exposure during high-altitude expedition cause psychomotor impairment. Neuroimaging studies indicated that the impairment may be significantly associated with neuron loss and decreased regional homogeneity $(\mathrm{ReHo})$ in several brain regions, suggesting the neural functionality in these regions may be utilized to predict psychomotor impairment under exposure. In this study, 69 subjects come from Shaanxi-Tibet immigrant cohort. Reaction time (RT) tasks were performed to measure the subject's psychomotor function before and after 2-year high-altitude exposure. For each individual, the RT differences between pre-exposure and post-exposure were calculated, which were referred to as "targets" in model establishment. Rs-fMRI data were acquired at the same time with RT tasks. For each individual, the map of ReHo alteration was generated, from which the patterns would be recognized. A pattern recognition procedure was utilized to train and test the predictive models. Two different cross-validation strategies were utilized to evaluate the model performance: leave-one-out cross-validation and four-fold cross-validation. For the models displaying significant $\mathbf{R}^{2}$ and MSE, weight maps were built. As a result, the predictive models were able to decode the changes of simple and recognition reaction time from the alterations of brain activation under the exposure. The regions with highest contributions to the predictions were bilateral putamen and bilateral pallidum, suggesting that predictions were mainly based on the patterns concentrated in these regions. This study was a proof of concept study designed to examine whether individual-level psychomotor impairment under highaltitude exposure could be predicted by a combination of pattern recognition approach and neuroimaging data.
\end{abstract}

Keywords: High altitude, psychomotor impairment, pattern recognition, regional homogeneity

Released online in J-STAGE as advance publication February $27,2019$.

${ }^{\S}$ These authors contributed equally to this work.

*Address correspondence to:

Prof. Wenjing Luo and Prof. Jingyuan Chen, Department of Occupational and Environmental Health, the Ministry of Education Key Lab of Hazard Assessment and Control in Special Operational Environment, School of Public Health, Air Force Medical University, 169 Changlexi Road, Xi'an, Shaanxi 710032 China.

E-mail: luowenj@fmmu.edu.cn (Luo WJ) jy_chen@fmmu.edu.cn (Chen JY)

\section{Introduction}

At high-altitude, hypoxia together with other physiological stressors, including low temperature, ultraviolet rays and dehydration, may lead to a decline in cognitive function (1). Previous studies have proved that acute and chronic hypoxia exposure during highaltitude expedition cause impairment in working memory, learning ability, attention and concentration. Recently, according to a previous study based on Tibet immigration population in China, two-year hypoxia 
exposure may lead to a significant impairment in psychomotor function, i.e., prolonged simple and recognition reaction time (2). Psychomotor function is related to daily affairs like driving a car, attending to conversation, tracking and responding to a set of simple instructions, whose impairment may cast a negative influence upon the life quality and work efficiency of high-altitude immigrants.

Neuroimaging approaches including high resolution 3D anatomical imaging and resting-state functional magnetic resonance imaging (rs-fMRI) have been employed to reveal the structural and functional basis of the hypoxia induced psychomotor impairment. Our previous work indicated the neuron loss and decreased regional homogeneity ( $\mathrm{ReHo}$ ) in several brain regions, i.e., putamen, superior temporal gyrus and anterior cingulate gyrus, may greatly contribute to the psychomotor impairment (2). ReHo was designed to reflect the similarity of the time series of a given voxel to those of its neighboring voxels, which may function as a potential sign of local neural functionality (3). Thus, we inferred that the alteration of regional neural activity may serve as a biomarker predicting the potential psychomotor impairment under highaltitude exposure and reflecting the individual's vulnerability to hypoxia stress. In clinical practice, the potential biomarker may be referred by physicians when they gave advices to voluntary high-altitude immigrants. In parallel, it also helps to understand the pathophysiological processes associated with these neurobehavioral alterations and provide biologicallyrelevant targets that can guide high-altitude impairment prevention and novel treatment development $(4,5)$.

Machine learning (ML) techniques can help to discover the potential biomarkers within the neuroimaging data. Compared with conventional univariate analyses, ML techniques are able to make regionally specific inferences about abnormalities in brain function associated with the impairment at the individual level (6). As a subfield of ML, pattern recognition approach uses computer-based techniques to automatically discover patterns in the data, which be utilized to identify relationships between patterns of brain activity and continuous measures of neurobehavior. In our case, ReHo data can be analyzed by pattern recognition approach to decode individual-level psychomotor measures (4).

The aim of this study was thus to use functional neuroimaging and pattern recognition approach to determine whether the change of psychomotor measures before and after high-altitude exposure could be decoded from patterns of ReHo alteration. This study has the potential to identify important biomarkers not only to help determine vulnerability to high-altitude hypoxia stress at the individual level, but also to pave the way forward for future studies using ML technique to predict other clinical outcomes in high-altitude immigrants.

\section{Materials and Methods}

\subsection{Subjects}

The protocol was approved by the Ethics Committee of the Medical Faculty of Fourth Military Medical University (registry no. KY20143344-1). All the studies were conducted in accordance with the ethical principles for medical research involving human subjects as defined in the Declaration of Helsinki.

All the subjects in this study come from ShaanxiTibet immigrant cohort (STI cohort). The details about this cohort have been described previously (2). The STI cohort study was launched in 2014, included 69 young healthy high school graduates in Shaanxi who were admitted into Tibet University for four-year higher education. Baseline investigation, neurobehavioral and MRI measures were performed on July, 2014 in Xi'an (altitude $466 \mathrm{~m}$ ), China. The information about high-altitude exposure history, medical history and sociodemographic status (parental education, vocation, socioeconomic status, etc.) were collected at baseline. The follow-up investigation and measures were performed on May 2016 in Lhasa (altitude 3,658 m).

Reaction time (RT) tasks were performed to measure the subject's psychomotor function before and after exposure, which consisted of visual simple reaction time (VSRT), audial simple reaction time (ASRT), visual recognition reaction time (VRRT) and audial recognition reaction time (ARRT). All the procedures were performed in the pattern of CNS Vital Signs (http://www.cnsvs.com/).

\subsection{Rs-fMRI data acquisition and analysis}

Rs-fMRI data were acquired with General Electric Discovery MR750 3.0T (General Electric Co. Ltd., Connecticut, USA) in Xijing Hospital of Air Force Medical University and the General Hospital of Tibet Military Region, respectively. The details about MRI scanning have been described previously (2). Data preprocessing was performed with the SPM8 and Data Processing Assistant for Resting-state fMRI Advanced (DPARSFA) tools as previously described $(2,7)$. The KCC (Kendall's coefficient concordance) value between the time series of a given voxel and those of its nearest 26 neighboring voxels was calculated in a voxel-wise method. Then, ReHo map for each individual was transformed to MNI coordinates and spatially smoothed (full width at half maximum $[\mathrm{FWHM}]=8 \mathrm{~mm}$ ). Finally, the ReHo map of each individual was divided by its own mean ReHo for standardization purposes.

\subsection{Predictive models establishment}

For each individual, the RT alteration during the exposure (RT post-exposure - RT pre-exposure) 
was calculated, which was referred to as "target" in the process of model establishment. Then, the ReHo difference between pre-exposure and post-exposure (ReHo post-exposure - ReHo pre-exposure) was calculated in the similar method used for calculating the RT alteration. For each individual, the map of ReHo differences was referred to as "input", from which the patterns would be recognized in the process of model establishment $(2,8)$.

A standard procedure in Pattern Recognition for Neuroimaging Toolbox (PRoNTo) (http://www.mlnl. cs.ucl.ac.uk/pronto/) was utilized to train and test the predictive models. The models were trained to learn the association between the ReHo and RT alterations using the examples in training sets. Three Pattern recognition algorithms currently available in PRoNTo: Relevance Vector Regression (RVR), Gaussian Process Regression (GPR) and Kernel Ridge Regression (KRR) were utilized to extract predictive patterns within the data of ReHo alterations (9). In the preliminary research, we found there were no significant differences in the performance of the three different algorithms. Herein, we only present the results of RVR for the sake of brevity. RVR is a probabilistic kernel-based pattern recognition method using Bayesian inference to obtain sparse regression models, and allows the extraction of patterns within a high-dimensional feature space, which was widely used in previous studies (10).

Two different cross-validation strategies were utilized to evaluate the model performance: leaveone-out cross-validation and 4-fold cross-validation. Leave-one-out cross validation is a frequently used validation method, involving leaving one subject out as the validation data, training the model on other $\mathrm{N}-1$ subjects, and doing so $\mathrm{N}$ times so that each subject is left out once. In 4-fold cross-validation, the original sample is randomly partitioned into 4 equal sized subsamples. Of the 4 subsamples, a single subsample is retained as the validation data, and the remaining 3 subsamples are used as training data (4).

In both cross-validation strategies, the RT alteration(s) left out for test was/were decoded from the corresponding data of ReHo alterations using the model trained on the remaining data. Agreement between predicted and actual RT alterations was assessed using two different metrics: coefficient of determination $\left(\mathrm{R}^{2}\right)$ and mean squared error (MSE). Statistical significance for both metrics was determined by permutation tests with 1000 times. Results were considered significant if the $p<0.05(4,9)$.

\subsection{Weight map}

For pattern recognition models showing significant $\mathrm{R}^{2}$ and MSE, weight maps were built at both voxel and region level. For each brain region defined by the Anatomical Automatic Labeling (AAL) atlas, the mean of all voxel weights (absolute values) within this region were computed. Then, all the labelled regions were ranked according to the percentage of the total normalized weights they contributed in the pattern recognition modelling $(4,9)$.

\section{Results and Discussion}

\subsection{Subjects' characteristics}

The average age of the subjects (M: 48; F: 21) was 18.2 \pm 0.3 (range: $17.5-19.1$ ) at baseline. In the 1 st year of follow-up, the cumulative high-altitude exposure time of the subjects was $270.0 \pm 7.4 \mathrm{~d}$ (range: $257-299 \mathrm{~d}$ ), while in the second year, the exposure time increased to $280.8 \pm 7.3 \mathrm{~d}$ (range: $268-314 \mathrm{~d}$ ). No major health problems occurred during the follow-up. The detailed demographic and socioeconomic information of subjects were reported in our previous study (2).

\subsection{Performance of the predictive model}

The correlations between the predicted RT alterations and the actual values were displayed in Figure 1. Coefficient of determination $\left(\mathrm{R}^{2}\right)$ and mean squared error (MSE) were calculated for each prediction to assess the goodness-of-fit. As a result, the $\mathrm{R}^{2}$ and MSE between the predicted and actual VSRT alteration were $0.33(p=0.003)$ and $0.01(p=0.001)$, respectively; Similar results were obtained in the prediction of ASRT alteration, the $\mathrm{R}^{2}$ and MSE were $0.46(p=0.001)$ and $0.01(p=0.001)$. The $\mathrm{R}^{2}$ and MSE between the predicted and actual VRRT alteration were 0.14 ( $p=$ $0.045)$ and $0.04(p=0.013)$, respectively; while the $\mathrm{R}^{2}$ and MSE between the predicted and actual ARRT alteration were $0.25(p=0.014)$ and $0.02(p=0.001)$. In summary, the predictive models were able to decode high-altitude induced psychomotor impairments from the changes of brain activation during the exposure.

\subsection{Regions contributed to prediction}

The weight maps of patterns contributed to predictions were displayed in Figure 2. The voxel-based weight maps were located on the left, while the regionbased weight maps (computed from the voxel based predictive pattern) were located on the right. The color of each region corresponds to the normalized average of voxels weights within the regions (in absolute value). The top 10 ranked regions according to normalized weights per region were displayed in Table 1 , which represent over $15 \%$ of the total weights in the decision functions. Generally, the regions with highest contributions to the predictions were bilateral putamen and bilateral pallidum, while Heschl's gyrus and cuneus gyrus also contributed to the predictions, suggesting that predictions were mainly based on the patterns 

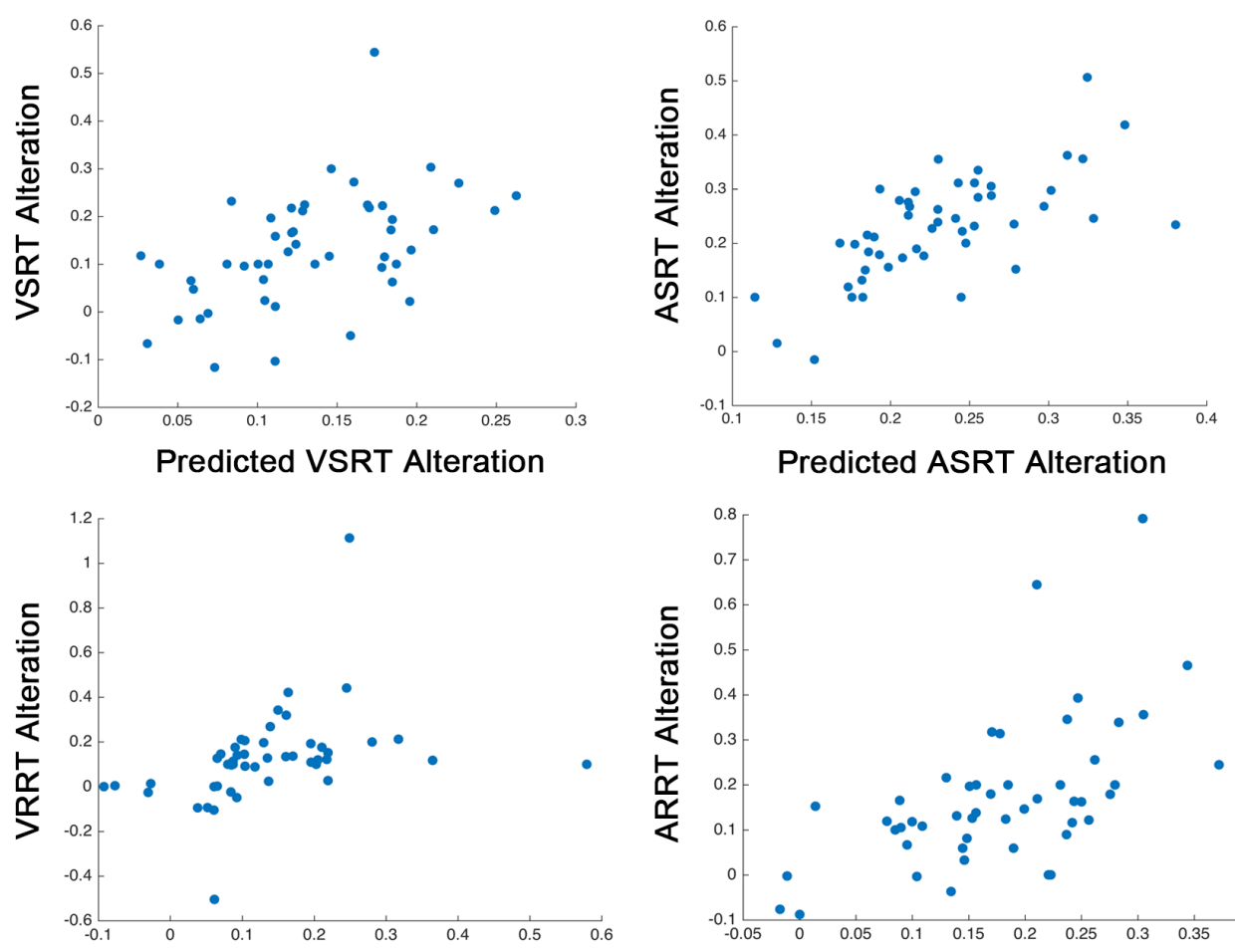

Predicted VRRT Alteration

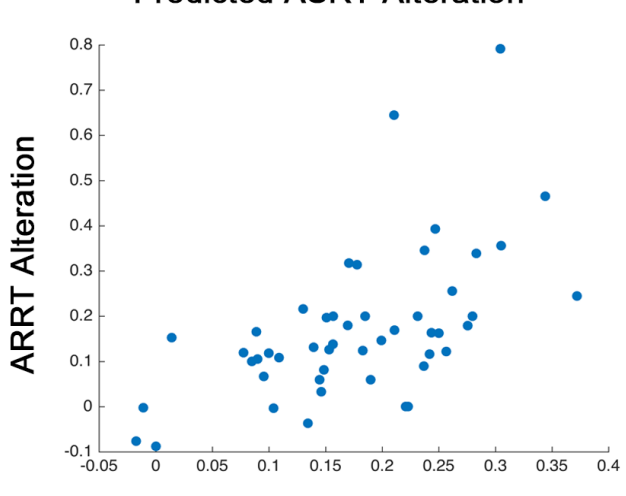

Predicted ARRT Alteration

Figure 1. The scatter plots displaying the predicted neurobehavioral alterations ( $\mathrm{x}$-axis) against the actual values or targets (y-axis). VSRT, visual simple reaction time; ASRT, audial simple reaction time; VRRT, visual recognition reaction time; ARRT, audial simple reaction time.

concentrated in these regions.

The neurobehavioral and rs-fMRI data used in the current study have been analyzed and reported in our previous study (2). Previously, we aimed at identifying the brain regions related to high-altitude induced cognitive impairment. Similar to other conventional neuroimaging studies, we utilized univariate statistical analysis to compare the neuroimaging data before exposure with those after exposure. The results showed significant associations between ReHo changes in putamen and alterations of the neurobehavioral alterations. However, a major limitation of the univariate analysis we used is that it only describes differences at the group level and does not enable decisions at the individual level, which is of more limited use in practice. In the present study, instead of investigating association between neurobehavioral measures and brain activity at the group level, we applied pattern recognition approach, with the aim of determining whether the alteration of neurobehavioral measures at different time points could be decoded from patterns of whole-brain activity changes. To the best of our knowledge, this is the first study applying pattern recognition approach to predict individual's cognitive impairment under high-altitude exposure.

The strength of this study was guaranteed the two different cross-validation strategies, four-fold cross-validation and leave-one-out cross-validation. Although it's common to leave one subject out and train the model with $\mathrm{N}-1$ subjects (where $\mathrm{N}$ corresponds to the total number of subjects), demonstration of reproducibility between different cross-validation strategies is vital to display the stability of the results and for future clinical use of the predictive model. The $\mathrm{R}^{2}$ and MSE between predicted and actual RT alterations were significant when the model was tested using the two different cross-validation strategies, suggesting the relationship between psychomotor function impairment and the brain activity alteration is reliable in the model establishment (9). Interestingly, we found the $\mathrm{R}^{2} \mathrm{~s}$ between predicted and actual VRRT/ ARRT alterations were lower than those in VSRT/ ASRT alteration prediction (MSEs were higher). One possible explanation for these findings is the fact that recognition reaction tasks included more brain regions and neurophysiological processes in responding to signals of complex directions, thus it's more difficult for the learning algorithm to learn the relationship between the neurobehavioral measures and brain activity.

Some brain areas are probably more informative about regression targets than others. The weight map is therefore a spatial representation of the decision function, i.e. every voxel within the mask contributes with a certain weight to the decision function. Since the prediction is based on the whole brain pattern, 
(A)
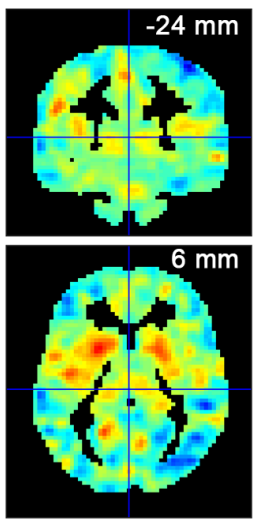

(B)
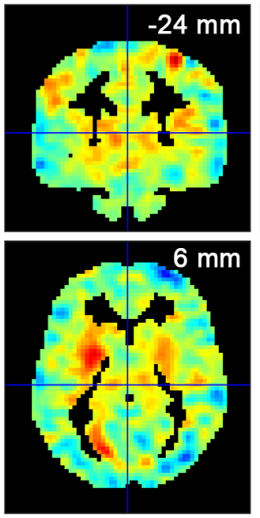

(C)
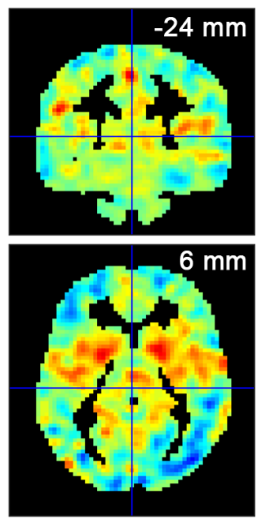

(D)
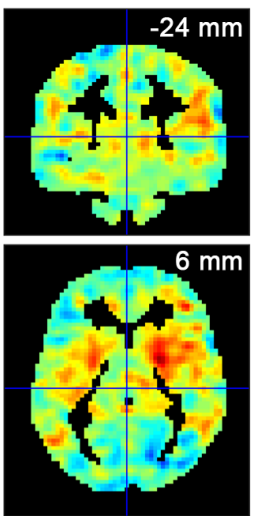
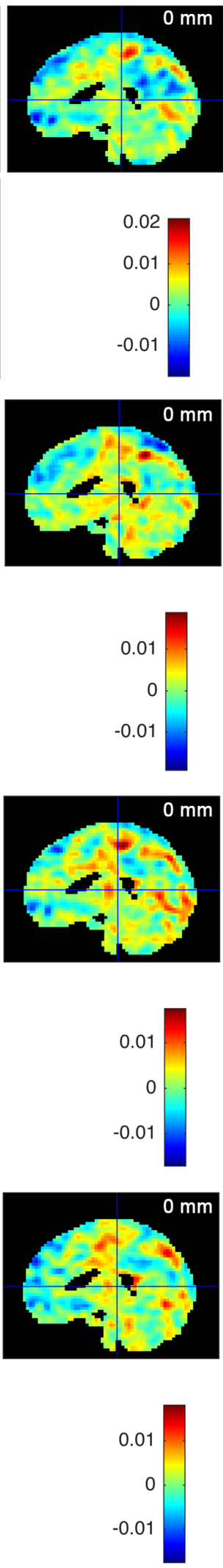
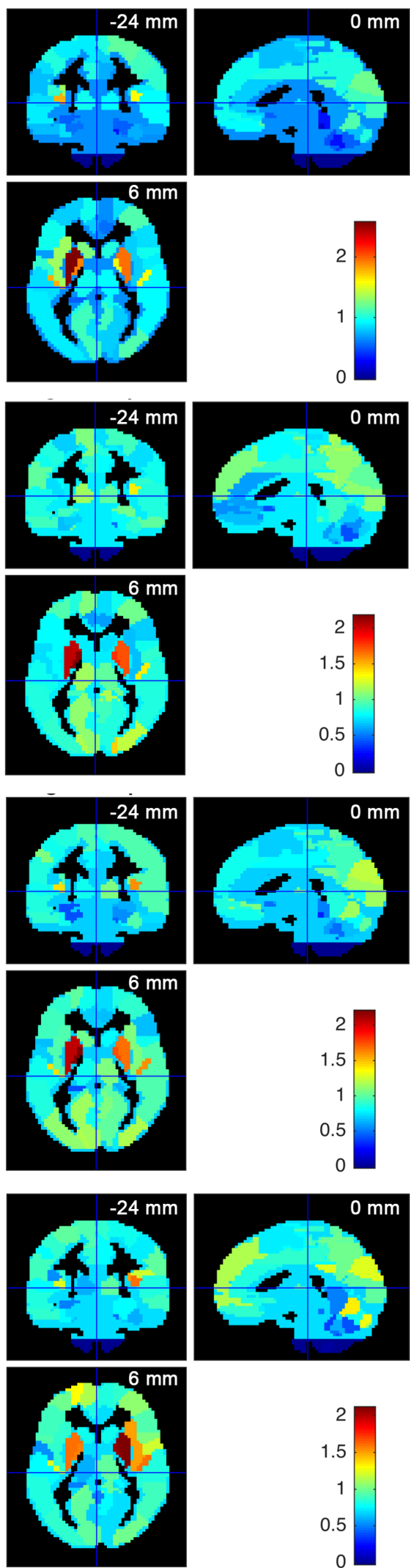

Figure 2. The weight map of patterns in the prediction of neurobehavioral alterations. (A) VSRT; (B) ASRT; (C) VRRT; (D) ARRT. The voxel-based weight maps were located on the left, and the color bar indicated the relative importance of the voxel in the decision function. The region-based pattern localization maps were located on the right, and the color bar indicated the normalized contribution of the region. 
Table 1. The top ten ranked regions contributed to the prediction of VSRT/ASRT/VRRT/ARRT alterations

\begin{tabular}{|c|c|c|}
\hline Regions & Weight (\%) & Size (voxels) \\
\hline \multicolumn{3}{|l|}{ Prediction of VSRT Alterations } \\
\hline L Putamen & 2.53 & 284 \\
\hline R Putamen & 1.87 & 318 \\
\hline L Pallidum & 1.82 & 79 \\
\hline L Heschl's Gyrus & 1.76 & 72 \\
\hline R Heschl's Gyrus & 1.51 & 60 \\
\hline R Pallidum & 1.47 & 68 \\
\hline L Insula & 1.27 & 560 \\
\hline R Gyrus Rectus & 1.24 & 185 \\
\hline L Angular Gyrus & 1.24 & 340 \\
\hline L Cuneus Gyrus & 1.22 & 460 \\
\hline \multicolumn{3}{|l|}{ Prediction of ASRT Alterations } \\
\hline L Pallidum & 2.18 & 79 \\
\hline L Putamen & 1.98 & 284 \\
\hline R Pallidum & 1.72 & 68 \\
\hline R Putamen & 1.63 & 318 \\
\hline R Superior Occipital Gyrus & 1.48 & 391 \\
\hline R Inferior Occipital Gyrus & 1.48 & 313 \\
\hline R Superior Frontal Gyrus, Orbital Part & 1.40 & 166 \\
\hline R Heschl's Gyrus & 1.31 & 60 \\
\hline R Middle Occipital Gyrus & 1.24 & 565 \\
\hline R Precuneus & 1.20 & 898 \\
\hline \multicolumn{3}{|l|}{ Prediction of VRRT Alterations } \\
\hline L Pallidum & 2.12 & 79 \\
\hline L Putamen & 1.98 & 284 \\
\hline R Pallidum & 1.68 & 68 \\
\hline R Heschl's Gyrus & 1.57 & 60 \\
\hline R Putamen & 1.57 & 318 \\
\hline L Heschl's Gyrus & 1.39 & 72 \\
\hline L Cuneus Gyrus & 1.21 & 460 \\
\hline L Angular Gyrus & 1.20 & 340 \\
\hline R Middle Frontal Gyrus, Orbital Part & 1.16 & 213 \\
\hline L Superior Occipital Gyrus & 1.15 & 353 \\
\hline \multicolumn{3}{|l|}{ Prediction of ARRT Alterations } \\
\hline R Putamen & 2.07 & 318 \\
\hline R Pallidum & 2.03 & 68 \\
\hline R Heschl's Gyrus & 1.60 & 60 \\
\hline L Putamen & 1.54 & 284 \\
\hline L Pallidum & 1.46 & 79 \\
\hline R Insula & 1.41 & 508 \\
\hline L Cerebellum Crus II & 1.33 & 518 \\
\hline L Superior Frontal Gyrus & 1.30 & 1,013 \\
\hline Vermis 6 & 1.29 & 87 \\
\hline L Cuneus Gyrus & 1.27 & 460 \\
\hline
\end{tabular}

The third column (weights) displayed the normalized contribution of each region. The rows of the table are sorted in descending order according to this value. The fourth column displayed the size of the patterns (voxels) in each region, indicating the overlap between the atlas and the data. VSRT, visual simple reaction time; ASRT, audial simple reaction time; VRRT, visual recognition reaction time; ARRT, audial simple reaction time.

rather than on individual regions or voxels, all voxels contribute to the regression and no conclusions should be drawn about a particular subset of voxels in isolation (4). The brain regions with the highest contribution to decoding the striatum, including the putamen and pallidum. This result is consistent with the results at group level in our previous study. The striatum is a critical component of the motor and reward systems, which receives glutamatergic and dopaminergic inputs from different sources and serves as the primary input to the rest of the basal ganglia. It is well established that movement disorders such as dyskinesias, chorea and choreoathetosis are associated with abnormal functioning within these regions (11-13). As a result, our findings suggest that neural functionality loss in these motor regulating regions may contribute to psychomotor impairment in high-altitude immigrants. Our investigation therefore indicates that these brain regions may provide critical information for successfully estimating vulnerability to high-altitude stress at the individual level.

There were some limitations in the present study. To be most important, although we applied two different cross-validation strategies to demonstrate generalizability of the predicative models, ideally the models should be tested with truly independent samples. Further studies with larger sample sizes are necessary to assess the generalizability of the proposed modelling approach by completely independent training and testing sets.

\section{Conclusion}

This study was designed to examine whether pattern recognition approach could be applied to neuroimaging data to predict individual-level psychomotor impairment under high-altitude exposure. Future studies, using a combination of pattern recognition and neuroimaging approaches, can build on the present findings to determine the individual's vulnerability to other cognitive impairments under long-term highaltitude exposure. Furthermore, as the follow-up of our STI cohort continues, we are trying to decode the cognitive impairment under long time highaltitude exposure (4-year or more) from the existing neuroimaging data acquired in relatively short time (2-year), whose findings may be more persuasive in advising the voluntary high-altitude immigrants in the clinical practice.

\section{Acknowledgement}

This study was financially supported by the National Science Foundation of China (No. 81330045, 81730053, $81803194,81502770)$ and the Military Logistics Research Project (No. AWS14L008, AWS16J022).

\section{References}

1. Yan X. Cognitive impairments at high altitudes and adaptation. High Alt Med Biol. 2014; 15:141-145.

2. Chen X, Zhang Q, Wang J, et al. Cognitive and neuroimaging changes in healthy immigrants upon relocation to a high altitude: A panel study. Hum Brain Mapp. 2017; 38:3865-3877.

3. Jiang L, Zuo XN. Regional homogeneity: A multimodal, multiscale neuroimaging marker of the human connectome. Neuroscientist. 2016; 22:486-505.

4. Schrouff J, Rosa MJ, Rondina JM, Marquand AF, Chu C, 
Ashburner J, Phillips C, Richiardi J, Mourão-Miranda J. PRoNTo: Pattern recognition for neuroimaging toolbox. Neuroinformatics. 2013; 11:319-337.

5. Taylor JA, Matthews N, Michie PT, Rosa MJ, Garrido MI. Auditory prediction errors as individual biomarkers of schizophrenia. Neuroimage Clin. 2017; 15:264-273.

6. Mateos-Perez JM, Dadar M, Lacalle-Aurioles M, Iturria-Medina Y, Zeighami Y, Evans AC. Structural neuroimaging as clinical predictor: A review of machine learning applications. Neuroimage Clin. 2018; 20:506522.

7. Yan CG, Zang YF. DPARSF: A MATLAB toolbox for "Pipeline" data analysis of resting-state fMRI. Front Syst Neurosci. 2010; 4:13.

8. Gualtieri CT, Johnson LG. Reliability and validity of a computerized neurocognitive test battery, CNS Vital Signs. Arch Clin Neuropsychol. 2006; 21:623-643.

9. Portugal LC, Rosa MJ, Rao A, et al. Can Emotional and Behavioral Dysregulation in Youth Be Decoded from Functional Neuroimaging? PloS One. 2016; 11:e0117603.

10. Ranlund S, Rosa MJ, de Jong S, Cole JH, Kyriakopoulos M, Fu CHY, Mehta MA, Dima D. Associations between polygenic risk scores for four psychiatric illnesses and brain structure using multivariate pattern recognition. Neuroimage Clin. 2018; 20:1026-1036.

11. Kandiah N, Tan K, Lim CC, Venketasubramanian N. Hyperglycemic choreoathetosis: role of the putamen in pathogenesis. Mov Disord. 2009; 24:915-919.

12. Singh-Bains MK, Waldvogel HJ, Faull RL. The role of the human globus pallidus in Huntington's disease. The role of the human globus pallidus in Huntington's disease. 2016; 26:741-751.

13. Nakawah MO, Lai EC. Post-stroke dyskinesias. Neuropsychiatr Dis Treat. 2016; 12:2885-2893.

(Received January 10, 2019; Revised February 3, 2019; Accepted February 9, 2019) 